\title{
Hypoxia Activates SUM0-1-HIF-1a Signaling Pathway to Upregulate Pro-inflammatory Cytokines and Permeability in Human Tonsil Epithelial Cells
}

Yan Lin

Shanghai university of Chinese traditional medicial

Mingjing Wang

Shanghai university of Chinese tradition medicine

Zhen Xiao

Shanghai university of Chinese tradition medicine

Zhiyan Jiang ( $\sigma_{\text {jzy_lh@163.com ) }}$

Shanghai university of Chinese traditional medical https://orcid.org/0000-0003-3213-177X

Original Article

Keywords: Adenoid hypertrophy, sleep disordered breathing, human tonsil epithelial cells, SUMO-1, HIF-1a, proinflammatory cytokines, M2 macrophages

Posted Date: February 4th, 2021

DOl: https://doi.org/10.21203/rs.3.rs-157614/v1

License: (9) This work is licensed under a Creative Commons Attribution 4.0 International License.

Read Full License 


\section{Abstract}

Adenoid hypertrophy $(\mathrm{AH})$ can cause harmful effects on untreated children, which include mouth breathing, chronic intermittent hypoxia, sleep disordered breathing (SDB), and even some behavioral problems. However, the molecular mechanisms underlying this pathophysiological process have remained poorly understood. In this study, with use of a variety of biochemical approaches including gene silencing and transiently ectopic protein expression, we examined the molecular effectors involved in this process in an in vitro model of human tonsil epithelial cells (HTECs). We found that a hypoxic condition caused a dramatic upregulation of SUMO-1 expression, a member of the ubiquitin-like protein family, which in turn stabilized hypoxia-inducible factor (HIF)-1 a by sumoylating this HIF subunit and thus preventing its ubiquitination and degradation in HTECs. We also found that activating HIF-1a promoted permeability of HTEC cells as well as production and secretion of a variety of proinflammatory cytokines including IL-6, IL-8, and TNF-a, and pro-angiogenic growth factor VEGF. Furthermore, our data showed that hypoxia-induced inflammation was markedly inhibited by M2 macrophages that possess potent anti-inflammatory function. Our results suggest that selectively inhibiting the SUMO-1-HIF-1a signaling pathway leads to inflammatory responses in human tonsil epithelial cells, which might be a novel therapeutic approach for managing hypoxia-induced SDB resulting from $\mathrm{AH}$.

\section{Introduction}

Adenoid hypertrophy $(\mathrm{AH})$ has been long considered as the most common cause of nasopharyngeal obstruction in children [17]. Multiple epidemiological studies have revealed that the prevalence of $\mathrm{AH}$ ranged from 19 to $38 \%$ in children between 3 and 9 years old $[1,6]$. Occurring in a chronic state, AH can persist for a long period and is always complicated with partial or complete impairment of nasal function, thus leading to mouth breathing to circumvent the limited passage of air through the nasopharynx and causing chronic intermittent hypoxia $(\mathrm{ClH})$ [1]. The most common clinical outcomes include sleep disordered breathing (SDB), which is a group of pathophysiological conditions characterized by abnormity in respiratory pattern during sleep because of such a hypoxic condition [14].

Hypoxia represents a condition under which the body is deprived of adequate oxygen supply at the tissue level [23]. An occurrence of hypoxia can cause a metabolic crisis and threaten physiological functions and cellular viability $[35,8]$. Eukaryotic cells have established an intrinsic and rapid oxygen-sensing system, known as hypoxia-inducible factors (HIFs), to facilitate hypoxic responses via HIF stabilization $[25,26]$. Furthermore, this system has been demonstrated to induce expression of more than 100 downstream target genes, thereby enhancing the oxygen supply and supporting anaerobic ATP generation [30]. With regards to the regulatory mechanisms of HIFs, it is well established that in the presence of oxygen, the subunit of this heterodimeric transcriptional factors is frequently subjected to proteasomal degradation while the $b$ subunit remains relatively stable [24]. Mechanistically, the oxygendependent degradation domain (ODDD) at the C-terminus of the HIF-1 a subunit controls degradation through prolyl hydroxylase domain (PHD) proteins, which in turn promote the interaction between HIF and von Hippel-Lindau-containing ubiquitin E3 ligase complex and subsequent ubiquitination and 
proteasomal degradation of HIF proteins [5]. Under hypoxia, the oxygen-requiring PHD and factor inhibiting HIF-1 a $(\mathrm{FIH})$ are inactivated, which leads to nuclear translocation of HIF-1a and activation of its target genes. Sumoylation is another common form of post-translational modification affecting the transcriptional activity of numerous transcription factors [7, 31, 13]. SUMO (Small Ubiquitin-related MOdifier) is a member of the ubiquitin-like protein family that includes four isoforms, SUMO-1, SUMO-2, SUMO-3, and SUMO-4. Indeed, a previous study has demonstrated that SUMO-1-mediated modification at Lys391/Lys477 residues of HIF-1a can significantly enhance this subunit's stability as well as transcriptional activity [3].

Mammalian cells possess sophisticated mechanisms to control pro-inflammatory responses, For example, microvascular endothelial cells have protective and barrier functions and interact with factors to mediate inflammatory responses [29]. Increased permeability is the most critical mechanism for edema, which is typically characterized by disruption of tight junctions (TJs) as well as cellular barrier $[16,10]$. TJs are heteromeric protein complex composed of occludin and various claudins. The normal barrier functions of epithelium are solely dependent on the integrity of TJs [33]. Many proinflammatory cytokines such as TNF-a, IL-1 $\beta \beta$, IL-6, have been identified in hypoxia [34]. At the cellular level, these mediators can cause TJs breakdown and hyperpermeability [36].

Macrophage polarization is a process by which macrophages are able to adopt to distinct functions in response to the signals from the surrounding microenvironment [2]. Such an ability is tightly linked to their functions in the organism: They not only serve as powerful effector cells of the innate immune system, but also play an important role in removal of cellular debris. A canonical dichotomic view classifies M1 macrophages as proinflammatory macrophages because of their ability of efficiently secreting varied types of proinflammatory cytokines and reactive oxygen species (ROS). In contrast, M2 macrophages are typically considered anti-inflammatory macrophages because of their powerful capability of repairing tissues [19]. By using human tonsil epithelial cells (HTECs) as an in vitro model, in this study, we sought to investigate the role of SUMO-1/HIF-1aa in induction of expression of proinflammatory cytokines and regulation of cell permeability, and further characterize anti-inflammatory functions of M2 macrophages in this pathophysiological process.

\section{Materials And Methods}

\section{Cell culture}

Human tonsil epithelial cells (HTEC) and macrophage U937 were obtained from cell bank of Shanghai Biology Institute (Shanghai, P.R. China) and cultured in DMEM media (Trueline, Kaukauna, WI, USA) supplemented with 10\% FBS (Thermo Fisher Scientific, USA), 2 mM L-glutamine, and 1\% penicillin/streptomycin (Solarbio, Beijing, P.R. China). Normoxic experiments at $37^{\circ} \mathrm{C}$ were carried out under normal atmospheric conditions ( $21 \% 02,5 \% \mathrm{CO} 2)$ while hypoxic experiments at $37^{\circ} \mathrm{C}$ were carried out with use of an anaerobic chamber (modified Anaerobic System Model 1029, Fisher Scientific ${ }^{\circledR}$,

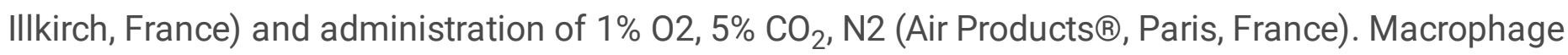


U397 cells were induced to differentiate into M2 type by using recombinant human IL-4 protein (P0002, Solarbio1, China).

\section{Enzyme-linked immunosorbent assay (ELISA)}

The concentrations of IL-6, IL-8 and TNF-a were determined using commercially available quantitative ELISA kits. All procedures were performed according to the protocol provided by the manufacturers. Briefly, the antibodies for IL-6IIL-8 and TNF-a were incubated at $37^{\circ} \mathrm{C}$ for $2 \mathrm{~h}$. Then, scrubbing solution was used to wash the ELISA plate for five times. After that, the secondary antibody was added. Finally, the stop solution was added to change the solution color from blue to yellow, and a microplate reader (Pulangxin, China) was used to determine the OD450 value within 5 minutes for each well. Triplicates were performed for all analysis.

\section{qRT-PCR}

Total RNAs from different samples were extracted by TRIzol Reagent (Invitrogen, Waltham, MA, USA). Then, RNAs were reverse transcribed into cDNA by cDNA synthesis kit (Thermo Fisher Scientific, Waltham, MA, USA) according to the manufacturer's instructions. The conditions of real-time PCR were as follows: $95^{\circ} \mathrm{C}$ for 10 minutes followed by 40 cycles of $95^{\circ} \mathrm{C}$ for 15 seconds and $60^{\circ} \mathrm{C}$ for 45 seconds. The relative expression was calculated by the $2-\Delta \Delta \mathrm{Ct}$ method with $U 6$ serving as the internal reference gene. All data represent the average of three replicates. The primers used in this study are listed as follows: SUMO1, F: 5'- GGGATAAGAAGGAAGGTG-3', R: 5'-CCAGTTCTTTTGGAGTATG-3'; IFNG, F: 5'CTCTTTTCTTAGGCATTTTG - 3', R: 5'-CGACAGTTCAGCCATCAC-3'; ARG1, F: 5'-CATAGGGATTATTGGAGC3', R: 5'-TTCTTCCGTTCTTCTTGAC-3'. GAPDH, F: 5'-AATCCCATCACCATCTTC-3', R: 5'AGGCTGTTGTCATACTTC - 3'.

\section{Western blot}

Whole protein lysates were extracted from different samples using RIPA lysis buffer (JRDUN, Shanghai, P.R. China) with EDTA-free Protease inhibitor Cocktail (Roche, Heidelberg, Germany). The concentration of protein samples was determined by an enhanced BCA protein assay kit (Thermo Fisher Scientific). Equal amounts of total protein $(25 \mathrm{mg}$ ) were fractionated on $10 \%$ SDS-PAGE and transferred to a nitrocellulose membrane (Millipore, Billerica, MA, USA) overnight. Then, after being blocked with $5 \%$ nonfat dry milk for 1 hour at room temperature, the membranes were probed at $4^{\circ} \mathrm{C}$ overnight with the primary antibodies followed by secondary antibody anti-mouse IgG (1:1,000; Beyotime, Shanghai, P.R. China) for 1 hour at $37^{\circ} \mathrm{C}$. An enhanced chemiluminescence system (Tanon, Shanghai, China) was used for detecting protein expression value. The information of primary antibodies is provided as follows: SUMO1 (ab133352, Abcam, UK), HIF-1a (ab16066, abcam, UK), VEGF (ab11934, Abcam, UK), occluding (ab216327, Abcam, UK), claudin-1(ab180158, Abcam, UK), ZO-1(ab190085, Abcam, UK) and GAPDH (\#5174, CST, USA). 


\section{Overexpression and knockdown of SUMO1 in HTEC cells}

Human SUM01 cDNA was cloned into the lentiviral core plasmid pLVX-Puro (Clontech, Palo Alto, CA, USA) to construct the recombinant plasmid pLVX-Puro-SUM01. The SUM01 targeting siRNA sequences (targeting sequence: siSUM01-1 (238-256): 5'- GCAGTGAGATTCACTTCAA-3'; siSUM01-2 (395-413): 5'GGAAGAAGATGTGATTGAA-3'; siSUM01-3 (727-745): 5'-GGCTTGTGGTGATAAATAA-3' were cloned into lentiviral core plasmid PLKO.1 (Addgene, Cambridge, MA, USA) to construct the recombinant plasmid pLKO.1-shARHGAP18. HTEC cells were pre-cultured in serum-free medium and co-transfected with liposome-mediated recombinant plasmid pLVX-Puro-SUMO1 and packaging plasmids psPAX2 and pMD2G.

\section{Cell permeability assay}

Briefly, Cells were inoculated into the upper chamber of the 24-well Transwell plate and cultured at $5 \%$ $\mathrm{CO}_{2}, 37^{\circ} \mathrm{C}$. The media was replaced daily. Then, fluorescently labeled dextran (FITC-Dextran, $1 \mathrm{mg} / \mathrm{mL}$ ) was added to culture media and subsequent cultured for $24 \mathrm{~h}$. The intensity of FITC fluorescence at 490 $\mathrm{nm}$ was measured using a microplate reader (Pulangxin, Beijing, China) and converted to permeability rate according to the standard curve.

Trans-epithelial/endothelial electrical resistance (TEER) assay

Millcell ERS-2 (Millicell ERS-2, Millipore, USA) was used to examine TEER values. All the procedures were performed according to the instructions of the manufacturer. The TEER value was calculated as follows: TEER value $(\Omega \cdot \mathrm{cm} 2)=$ TEER $(\Omega) \times$ surface area $\left(0.6 \mathrm{~cm}^{2}\right)$. Triplicate replications were performed for each experiment.

\section{Co-immunoprecipitation (Co-IP) assay}

Whole cell extracts were prepared after transfection or stimulation with appropriate ligands, followed by incubation overnight with the appropriate antibodies plus Protein A/G beads (Santa Cruz Biotechnology, USA). Beads were washed five times and separated by SDS-PAGE. Western blot was performed by using the antibodies as indicated above.

\section{Ubiquitination assay}

HTEC cells were transfected with non-specific siRNAs or siSUM01. Then, the cells were lysed in $1 \%$ SDScontaining radio immunoprecipitation assay (RIPA) buffer by sonication on ice. Then, cell lysates were treated by Protein A/G PLUS-Agarose (sc-2003, Santa Cruz Biotechnology, USA) for $1 \mathrm{~h}$. After that, each sample was incubated with the lgG (sc-2027, Santa Cruz Biotechnology, USA) overnight at $4^{\circ} \mathrm{C}$. Then, the nuclear pellets were collected by centrifugation at $3000 \mathrm{rpm}$ for $5 \mathrm{~min}$ at $4^{\circ} \mathrm{C}$ and subsequently washed 
by Protein A/G Plus-Agarose beads for four times. The purified proteins were separated by $4-20 \%$ gradient SDS-PAGE. Anti-HIF-1a antibody (ab1, Abcam, UK) and anti-Ubiquitin antibody (ab7780, Abcam, UK) were used for immunoblotting.

\section{Flow cytometry assay}

U937 macrophages were re-suspended with FACS buffer (PBS supplemented with $0.2 \%$ BSA, $0.01 \%$ $\mathrm{NaN}_{3}$ ) and stained with mAbs against CD86 and CD163, and isotype-matched IgG (obtained from BioLegend) for $30 \mathrm{~min}$ at $4^{\circ} \mathrm{C}$, after preincubation with rabbit IgG (obtained from Sigma) to block nonspecific bindings. Four-color flow cytometric analysis was carried out using the following Abs: antihuman CD163 FITC (563697, BD, USA) and anti-Mouse CD86 FITC (553691, BD, USA). Fluorescence was quantitated on a FACSCalibur flow cytometer equipped with CellQuest software (BD-Biosciences). Cells were gated according to their light-scatter properties to exclude cell debris.

\section{Statistical analysis}

GraphPad Prism software Version 7.0 (La Jolla CA, USA) was used for statistical analyses. Data are displayed as mean \pm SD for at least three replicates. Comparison between two groups was performed using Student t test, while comparison among multiple groups was performed using One-Way Analysis of Variance. A P-value $<0.05$ indicates statistical significance.

\section{Results}

\section{Hypoxia upregulates expression of SUMO-1 in human tonsil epithelial cell}

Previous study has shown that SUMO-1 can increase the stability and transcriptional activity of HIF-1aa [3], we first tested the effects of hypoxia on expression of SUMO-1 in HTECs. We treated HTECs for different time points as indicated, and found that hypoxia caused an increase in expression of SUMO-1 at both mRNA (Fig. 1A) and protein (Fig. 1B) levels in a time-dependent manner. These data suggest that hypoxia treatment enhances SOMO-1 expression in human tonsil epithelial cells.

\section{SUM0-1 promotes permeability of human tonsil epithelial cells}

We then performed siRNA-mediated gene silencing and protein overexpression to explore the relationship between SUMO-1 and cell permeability in HTECs. The silencing effects of short interfering RNA (siRNAs) targeting SUMO-1 were shown in Fig. 2A and 2B, while SUMO-1 overexpression was validated as shown in Fig. $2 \mathrm{C}$ and 2D. 
We next assessed changes in levels of proinflammatory cytokines in response to silencing or overexpression of SUMO-1 in HTECs. As shown in Fig. 3A, hypoxia caused a significant increase in secretions of IL-6, IL-8, and TNF-a, but the depletion of SUMO-1 attenuated such stimulatory effects.

Hyperpermeability usually results from a disruption of TJs and undermined integrity of endothelial barriers [32]. Here we found that the hypoxia treatment caused a significant decrease in transepithelial/endothelial electrical resistance (TEER) (Fig. 3B), but a significant increase in fluorescein isothiocyanate (FITC) - dextran staining (Fig. 3C). Both effects were reversed when SUMO-1 was silenced, thereby indicating a positive correlation between abundance of SUMO-1 and permeability of HTECs.

The integrity of TJs is dependent on formation of multiprotein complexes among integral membrane proteins such as claudins and occludin, and cytoplasmic scaffolding proteins, including zonula occludens (ZO)-1, ZO-2 and ZO-3 [4]. As shown in Fig. 3D, an exposure of HTECs to hypoxia led to a decrease in protein levels of ZO-1, Occludin, and Claudin-1, but a concurrent increase of SUMO-1, HIF-1aa, and pro-angiogenic growth factor VEGF in HTECs.

Collectively, these data support that hypoxia induces hyperpermeability of HTECs by upregulating activity of SUMO-1 and HIF-1aa to disrupt the integrity of TJs and cellular barrier.

\section{HIF-1a is sufficient for inducing hyperpermeability in human tonsil epithelial cells}

We further investigated the role of HIF-1a in contributing to hyperpermeability in HTECs. As shown in Fig. 4A, overexpression of SUMO-1 caused significantly increased secretion of IL-6, IL-8, and TNF-a. However, such effects were reversed when HTECs were treated with 2-methoxyestradiol (2-MeOE2), a potent inhibitor of HIF-1 a activity and inflammatory diseases [22, 12]. In consistent, 2-MeOE2 treatment also abolished SUMO-1 overexpression-induced hyperpermeability of HTECs, as reflected by changes in ZO-1, Occludin, and Claudin-1 levels (Fig. 4B-4D). These data support that HIF-1aa plays a critical role in contributing to hypoxia/SUMO-1-induced hyperpermeability in human tonsil epithelial cells.

\section{SUM0-1 interacts HIF-1a in human tonsil epithelial cells}

To investigate whether HIF-1a is regulated by SUMO-1, we used co-immunoprecipitation assay to identify a physical interaction between SUMO-1 and HIF-1 aa in HTECs (Fig. 5). These data suggest that SUMO-1 directly interacts with HIF-1 aa to cause sumoylation and in turn prevent its proteasomal degradation in HTECS.

\section{M2 macrophages prevent hypoxia-induced proinflammatory reaction in human tonsil epithelial cells}


We finally determined the role of M2 macrophages in inhibition of hypoxia-mediated inflammation in HTECs. IL-4 is a potent inducer for M2 macrophage polarization and differentiation (Supplementary Fig. 1), consistent with previous studies [27]. We found that a co-culture of IL-4-induced M2 macrophages and HTECs significantly attenuated hypoxia-induced upregulation of proinflammatory cytokines IL-6, IL-8, and TNF-a (Fig. 6A) and permeability (Fig. 6B and 6C) of HTECs. In addition, we also observed remarkably repressed levels of SUMO-1, HIF-1 aa, and VEGF, but enhanced expression levels of ZO-1, Occludin, and Claudin-1 in HTECs co-cultured with IL-4-induced M2 macrophages (Fig. 6D). Collectively, these data support that M2 macrophages play an important role in controlling hypoxia-induced inflammation in HTECs.

\section{Discussion}

$\mathrm{AH}$ is a serious health concern because of its possible deleterious effects on untreated children. $\mathrm{AH}-$ caused alterations in face development and impairment of nasal function may ultimately lead to mouth breathing, $\mathrm{ClH}$ and SDB, which are sometimes even complicated with behavioral problems $[9,21]$. Still, the mechanisms underlying the relationship between $\mathrm{AH}$ and hypoxia/SDB have remained poorly investigated. In this study, with use of human HTECs as an in vitro model, we have found that hypoxia caused upregulation of SUMO-1, which subsequently stabilized HIF-1 aa by preventing its degradation. This in turn enhanced the hyperpermeability of HTEC cells and the secretion of a variety of proinflammatory cytokines including IL-6, IL-8, and TNF-a, and pro-angiogenic growth factor VEGF. In addition, our data also support that hypoxia-induced tissue injury can be repaired by M2 macrophages. Therefore, our data have established a molecular pathway linking hypoxia with initiation of inflammation and hypertrophy of tonsils in $\mathrm{AH}$.

Here we reported that SUMO-1 gene expression is augmented by a hypoxic stimulation in human tonsil epithelial cells. Consistent with our in vitro findings, both mRNA and protein levels of SUMO-1 have been shown to be increased by hypoxic stimulation in a variety of cultured cell lines and in vivo, such as in mouse brain and heart $[11,28]$. The significant increases in levels of SUMO-1 mRNAs and proteins after hypoxic stimulation observed in human tonsil epithelial cells suggest a role of SUMO-1 in mediating hypoxic response in $\mathrm{AH}$ tissues.

We have also demonstrated that SUMO-1 upregulated protein levels and transcriptional activity of HIF-1a in human tonsil epithelial cells. Previous studies have indicated that SUMO-1 can conjugate to a number of functionally diverse proteins involved in the post-translational modification process of sumoylation $[18,20]$. In the present study, we observed that the protein levels of HIF-1a were markedly increased in the presence of overexpressed SUMO-1, but reduced when SUMO-1 was silenced under a hypoxic condition. In addition, proinflammatory cytokines IL-6, IL-8, and TNF- $a$ and pro-angiogenic mitogen and growth factors, such as VEGF, were increased with overexpression of SUMO-1 but decreased with its depletion, which are consistent with previous results showing that SUMO-1 was upregulated by hypoxia to interact with HIF-1a to result in its sumoylation, protecting HIF-1aa from ubiquitination and degradation $[3,28]$. Stabilized HIF-1a localizes in the nucleus and contributes to transcriptional activation of downstream 
genes. Coupled with concurrent decreases in levels of proteins essential for maintenance of tight junctions and cellular barrier function, these data further suggest that activation of SUMO-1/ HIF-1aa signaling plays a critical role in contributing to hyperpermeability of human tonsil tissues under the hypoxic condition.

Our data highlight an important role of macrophage polarization towards M2 in controlling hypoxiacaused tonsil injury in AH. Macrophages possess a remarkable plasticity in response to environmental changes and are able to switch between different phenotypes and exert distinct functions [15].

Macrophages can be grouped into two major phenotypes: An M1 phenotype that is induced by microbial agents including lipopolysaccharides (LPS), characterized by proinflammatory features, and lead to a Type 1 response; and M2 phenotype that is usually induced by IL-4, IL-13 or IL-10, characterized by antiinflammatory features, and initiate a Type 2 response. Here we observed that co-culture of IL-4-induced M2 macrophages with HTECs led to a significant inhibition of hypoxia-induced inflammation in HTECs. Thus, our study provides additional evidence for the anti-inflammatory function of M2 macrophages.

\section{Conclusion}

In summary, we report a SUMO-1/ HIF-1aa regulatory pathway contributing to hypoxia-induced tissue injury in $\mathrm{AH}$. Our results suggest that selectively inhibiting this pathway in human tonsil epithelial cells might be a novel therapeutic approach for controlling hypoxia-induced SDB. Our future directions would include testing this hypothesis in vivo, such as in rodent models, to validate its therapeutic effects.

\section{Declarations}

\section{Funding}

This research was financially supported by the National Natural Science Foundation of China (No. 81804143) and Longhua Hospital Science and Technology Innovation project (No.KY1928)

\section{Conflict of interest}

The authors declared that no conflict of interest.

\section{Ethics approval}

Not applicable

\section{Consent to participate}

Not applicable 


\section{Consent for publication}

Not applicable

\section{Availability of data and material}

The datasets used during the current study are available from the corresponding author on reasonable request.

\section{Code availability}

Not applicable

\section{Authors' contributions}

Zhiyan Jiang designed this project and revised the manuscript; Yan Lin performed the experiments and wrote the draft; Mingjing Wang analyzed the data and edited diagrams. Zhen Xiao help to technical assistance. All authors have contributed to read and agreed the final content of manuscript for submission.

\section{References}

1. Abreu, R. R., R. L. Rocha, J. A. Lamounier, and A. F. Guerra. 2008. Etiology, clinical manifestations and concurrent findings in mouth-breathing children. J Pediatr (Rio J) 84 (6):529-535. doi:10.2223/JPED.1844.

2. Atri, C., F. Z. Guerfali, and D. Laouini. 2018. Role of Human Macrophage Polarization in Inflammation during Infectious Diseases. Int J Mol Sci 19 (6). doi:10.3390/ijms19061801.

3. Bae, S. H., J. W. Jeong, J. A. Park, S. H. Kim, M. K. Bae, S. J. Choi, and K. W. Kim. 2004. Sumoylation increases HIF-1 alpha stability and its transcriptional activity. Biochem Biophys Res Commun 324 (1):394-400. doi:10.1016/j.bbrc.2004.09.068.

4. Bauer, H., J. Zweimueller-Mayer, P. Steinbacher, A. Lametschwandtner, and H. C. Bauer. 2010. The dual role of zonula occludens (ZO) proteins. J Biomed Biotechnol 2010:402593. doi:10.1155/2010/402593.

5. Berta, M. A., N. Mazure, M. Hattab, J. Pouyssegur, and M. C. Brahimi-Horn. 2007. SUMOylation of hypoxia-inducible factor-1alpha reduces its transcriptional activity. Biochem Biophys Res Commun 360 (3):646-652. doi:10.1016/j.bbrc.2007.06.103.

6. Bitar, M. A., G. Birjawi, M. Youssef, and N. Fuleihan. 2009. How frequent is adenoid obstruction? Impact on the diagnostic approach. Pediatr Int 51 (4):478-483. doi:10.1111/j.1442- 
200X.2008.02787.x.

7. Bossis, G., and F. Melchior. 2006. Regulation of SUMOylation by reversible oxidation of SUMO conjugating enzymes. Mol Cell 21 (3):349-357. doi:10.1016/j.molcel.2005.12.019.

8. Brahimi-Horn, M. C., J. Chiche, and J. Pouyssegur. 2007. Hypoxia signalling controls metabolic demand. Curr Opin Cell Biol 19 (2):223-229. doi:10.1016/j.ceb.2007.02.003.

9. Brambilla, I., A. Pusateri, F. Pagella, D. Caimmi, S. Caimmi, A. Licari, S. Barberi, A. M. Castellazzi, and G. L. Marseglia. 2014. Adenoids in children: Advances in immunology, diagnosis, and surgery. Clin Anat 27 (3):346-352. doi:10.1002/ca.22373.

10. Chen, L. W., W. J. Chang, J. S. Wang, and C. M. Hsu. 2006. Thermal injury-induced peroxynitrite production and pulmonary inducible nitric oxide synthase expression depend on JNK/AP-1 signaling. Crit Care Med 34 (1):142-150. doi:10.1097/01.ccm.0000190621.48720.8c.

11. Comerford, K. M., M. O. Leonard, J. Karhausen, R. Carey, S. P. Colgan, and C. T. Taylor. 2003. Small ubiquitin-related modifier-1 modification mediates resolution of CREB-dependent responses to hypoxia. Proc Natl Acad Sci U S A 100 (3):986-991. doi:10.1073/pnas.0337412100.

12. Dai, Y., M. Xu, Y. Wang, Z. Pasha, T. Li, and M. Ashraf. 2007. HIF-1Aalpha induced-VEGF overexpression in bone marrow stem cells protects cardiomyocytes against ischemia. J Mol Cell Cardiol 42 (6):1036-1044. doi:10.1016/j.yjmcc.2007.04.001.

13. Gill, G. 2005. Something about SUMO inhibits transcription. Curr Opin Genet Dev 15 (5):536-541. doi:10.1016/j.gde.2005.07.004.

14. Hosselet, J., I. Ayappa, R. G. Norman, A. C. Krieger, and D. M. Rapoport. 2001. Classification of sleepdisordered breathing. Am J Respir Crit Care Med 163 (2):398-405. doi:10.1164/ajrccm.163.2.9808132.

15. Jetten, N., S. Verbruggen, M. J. Gijbels, M. J. Post, M. P. De Winther, and M. M. Donners. 2014. Antiinflammatory $\mathrm{M} 2$, but not pro-inflammatory M1 macrophages promote angiogenesis in vivo. Angiogenesis 17 (1):109-118. doi:10.1007/s10456-013-9381-6.

16. Liu, J., J. Liu, H. Wang, and M. Bai. 2019. Protective effect of celastrol for burn-induced acute lung injury in rats. Int J Clin Exp Pathol 12 (2):576-583.

17. Major, M. P., H. Saltaji, H. El-Hakim, M. Witmans, P. Major, and C. Flores-Mir. 2014. The accuracy of diagnostic tests for adenoid hypertrophy: a systematic review. J Am Dent Assoc 145 (3):247-254. doi:10.14219/jada.2013.31.

18. Melchior, F. 2000. SUMO-nonclassical ubiquitin. Annu Rev Cell Dev Biol 16:591-626. doi:10.1146/annurev.cellbio.16.1.591.

19. Mills, C. D., K. Kincaid, J. M. Alt, M. J. Heilman, and A. M. Hill. 2000. M-1/M-2 macrophages and the Th1/Th2 paradigm. J Immunol 164 (12):6166-6173. doi:10.4049/jimmunol.164.12.6166.

20. Muller, S., C. Hoege, G. Pyrowolakis, and S. Jentsch. 2001. SUMO, ubiquitin's mysterious cousin. Nat Rev Mol Cell Biol 2 (3):202-210. doi:10.1038/35056591. 
21. Pereira, L., J. Monyror, F. T. Almeida, F. R. Almeida, E. Guerra, C. Flores-Mir, and C. Pacheco-Pereira. 2018. Prevalence of adenoid hypertrophy: A systematic review and meta-analysis. Sleep Med Rev 38:101-112. doi:10.1016/j.smrv.2017.06.001.

22. Pribluda, V. S., E. R. Gubish, Jr., T. M. Lavallee, A. Treston, G. M. Swartz, and S. J. Green. 2000. 2Methoxyestradiol: an endogenous antiangiogenic and antiproliferative drug candidate. Cancer Metastasis Rev 19 (1-2):173-179. doi:10.1023/a:1026543018478.

23. Schodel, J., and P. J. Ratcliffe. 2019. Mechanisms of hypoxia signalling: new implications for nephrology. Nat Rev Nephrol 15 (10):641-659. doi:10.1038/s41581-019-0182-z.

24. Schofield, C. J., and P. J. Ratcliffe. 2005. Signalling hypoxia by HIF hydroxylases. Biochem Biophys Res Commun 338 (1):617-626. doi:10.1016/j.bbrc.2005.08.111.

25. Semenza, G. L. 1998. Hypoxia-inducible factor 1: master regulator of 02 homeostasis. Curr Opin Genet Dev 8 (5):588-594. doi:10.1016/s0959-437x(98)80016-6.

26. Semenza, G. L. 1999. Regulation of mammalian 02 homeostasis by hypoxia-inducible factor 1. Annu Rev Cell Dev Biol 15:551-578. doi:10.1146/annurev.cellbio.15.1.551.

27. Shahbazi, M. A., M. Sedighi, T. Bauleth-Ramos, K. Kant, A. Correia, N. Poursina, B. Sarmento, J. Hirvonen, and H. A. Santos. 2018. Targeted Reinforcement of Macrophage Reprogramming Toward M2 Polarization by IL-4-Loaded Hyaluronic Acid Particles. ACS Omega 3 (12):18444-18455. doi:10.1021/acsomega.8b03182.

28. Shao, R., F. P. Zhang, F. Tian, P. Anders Friberg, X. Wang, H. Sjoland, and H. Billig. 2004. Increase of SUMO-1 expression in response to hypoxia: direct interaction with HIF-1Aalpha in adult mouse brain and heart in vivo. FEBS Lett 569 (1-3):293-300. doi:10.1016/j.febslet.2004.05.079.

29. Swerlick, R. A., and T. J. Lawley. 1993. Role of microvascular endothelial cells in inflammation. J Invest Dermatol 100 (1):111S-115S. doi:10.1111/1523-1747.ep12356595.

30. Taylor, C. T. 2008. Mitochondria, oxygen sensing, and the regulation of HIF-2alpha. Focus on "Induction of HIF-2alpha is dependent on mitochondrial 02 consumption in an O2-sensitive adrenomedullary chromaffin cell line". Am J Physiol Cell Physiol 294 (6):C1300-1302. doi:10.1152/ajpcell.00206.2008.

31. Tempe, D., M. Piechaczyk, and G. Bossis. 2008. SUMO under stress. Biochem Soc Trans 36 (Pt 5):874-878. doi:10.1042/BST0360874.

32. Vermette, D., P. Hu, M. F. Canarie, M. Funaro, J. Glover, and R. W. Pierce. 2018. Tight junction structure, function, and assessment in the critically ill: a systematic review. Intensive Care Med Exp 6 (1):37. doi:10.1186/s40635-018-0203-4.

33. Wittekindt, O. H. 2017. Tight junctions in pulmonary epithelia during lung inflammation. Pflugers Arch 469 (1):135-147. doi:10.1007/s00424-016-1917-3.

34. Ye, J., Z. Gao, J. Yin, and Q. He. 2007. Hypoxia is a potential risk factor for chronic inflammation and adiponectin reduction in adipose tissue of ob/ob and dietary obese mice. Am J Physiol Endocrinol Metab 293 (4):E1118-1128. doi:10.1152/ajpendo.00435.2007.

35. Yeo, E. J. 2019. Special issue on hypoxia. Exp Mol Med 51 (6):1-3. doi:10.1038/s12276-019-0257-8. 
36. Youn, Y. K., C. Lalonde, and R. Demling. 1992. Oxidants and the pathophysiology of burn and smoke inhalation injury. Free Radic Biol Med 12 (5):409-415. doi:10.1016/0891-5849(92)90090-4.

\section{Figures}

A

HTEC

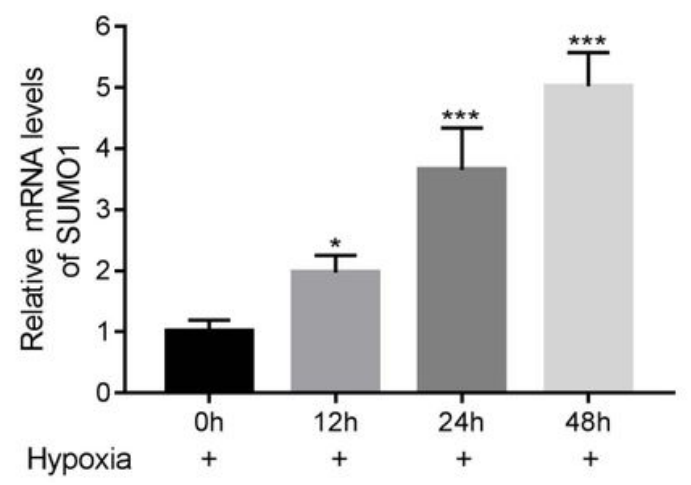

B

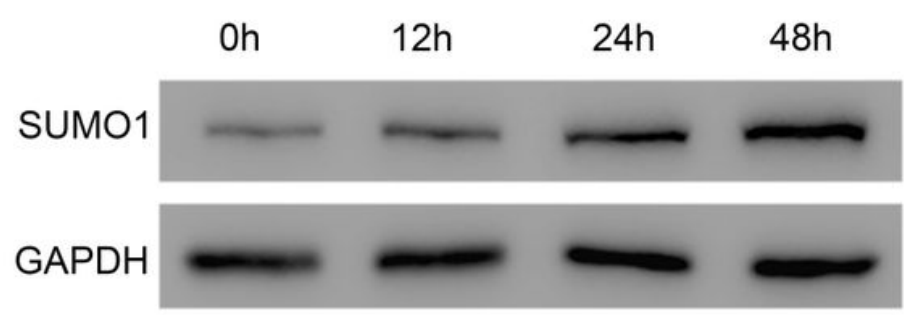

\section{Figure 1}

SUMO-1 is upregulated in a time-dependent manner in HTEC cells under hypoxic condition. RT-qPCR and Western Blotting assays were used to examine the relative mRNA (A) and protein levels (B) of SUMO1 in HTECs at $0 \mathrm{~h}, 12 \mathrm{~h}, 24 \mathrm{~h}$ and $48 \mathrm{~h}$ with hypoxia condition. *** $\mathrm{p}<0.001$ vs $0 \mathrm{~h}$. 
A

B

HTEC

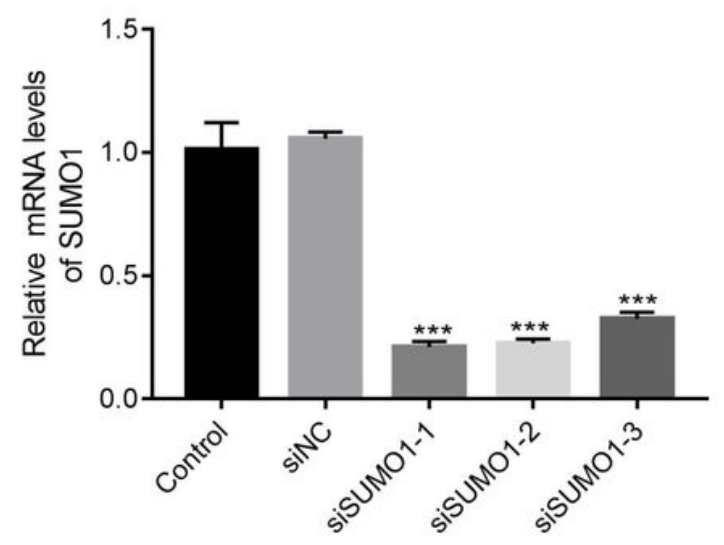

C

HTEC

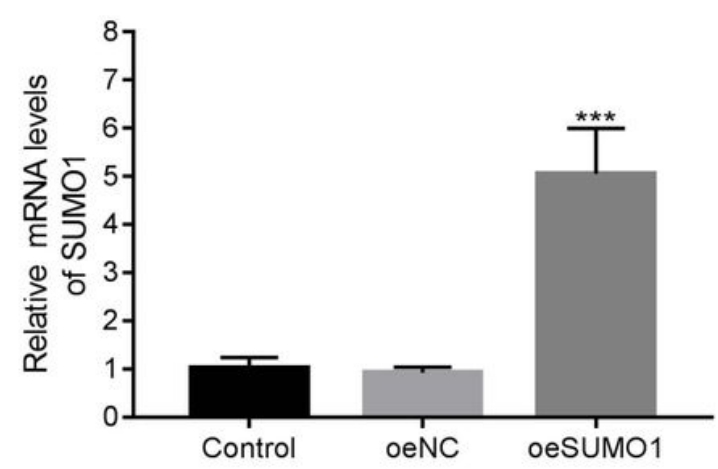

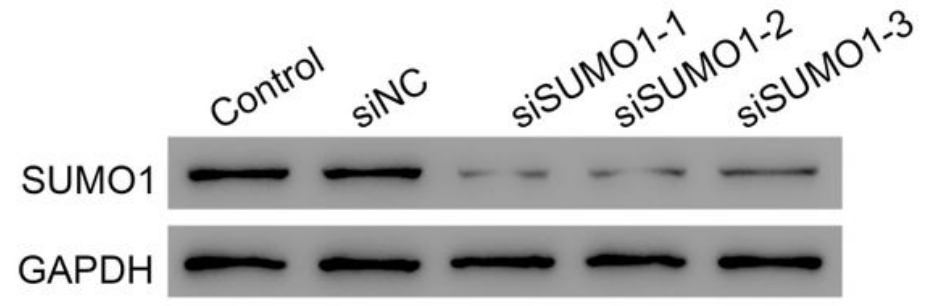

D

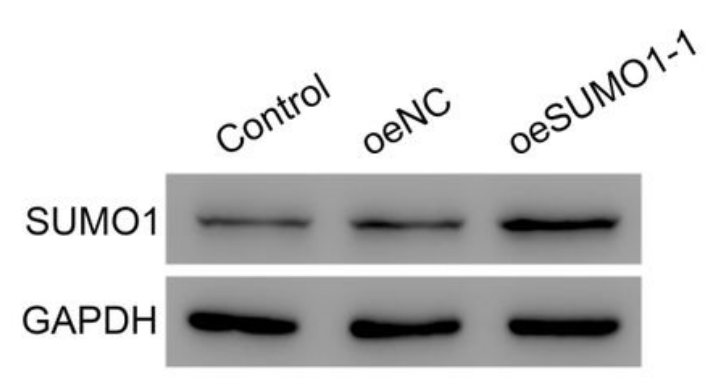

Figure 2

Knockdown and overexpression of SUMO1 in HTEC cells. (A) and (B) The relative mRNA and protein levels of SUMO1 were significantly suppressed in HTEC cells transfected with siSUM01-1, siSUM01-2 and siSUM01-3, respectively. ${ }^{* \star *} p<0.001$ vs siNC. (C) and (D): The relative mRNA and protein levels of SUM01 were determined in HTEC cells induced for overexpression of SUMO-1 using lentiviral vectors. *** $p<0.001$ vs oeNC. 
A

HTEC

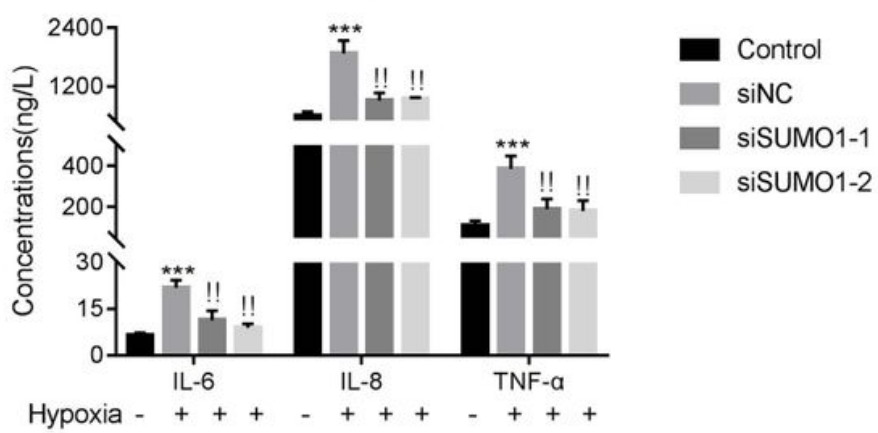

C

HTEC

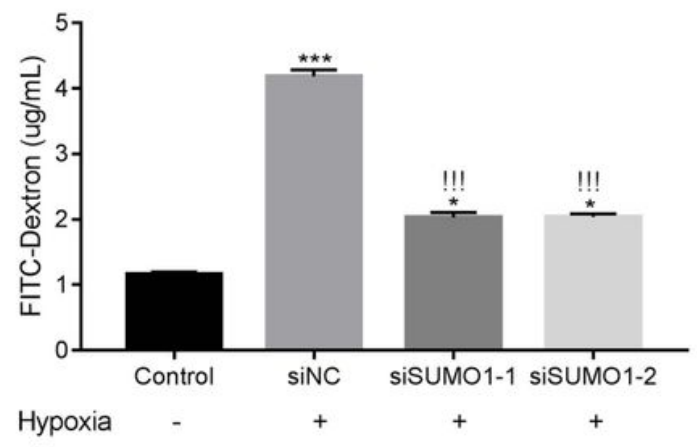

B

HTEC

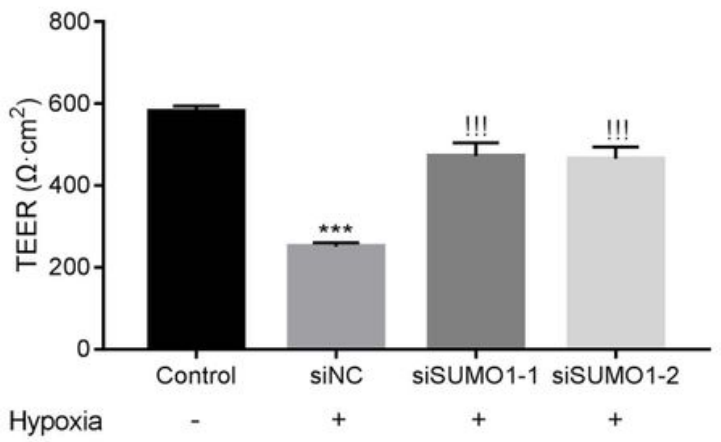

D

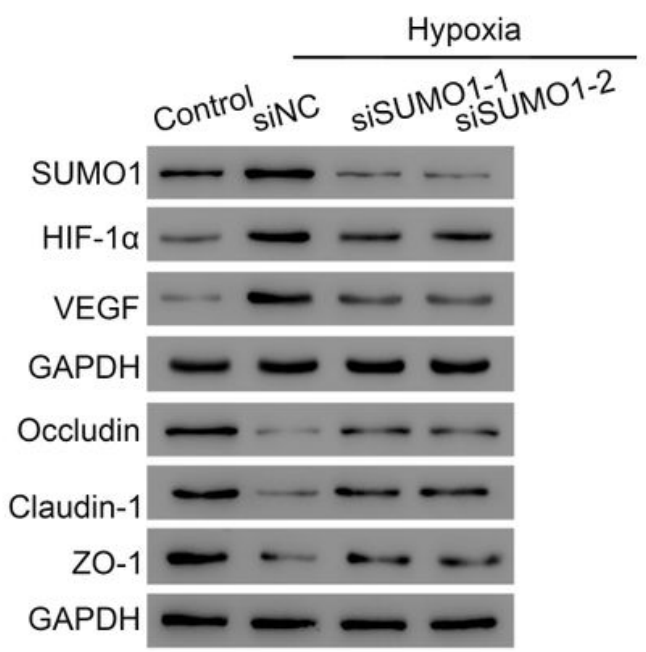

\section{Figure 3}

SUM01 silencing contributes to reduction in permeability of HTEC cells under hypoxic condition. (A) Knockdown of SUMO1 inhibited the secretion of IL-6, IL-8 and TNF- $a$ in HTEC cells treated under hypoxic condition for $24 \mathrm{~h}$. ${ }^{* \star} \mathrm{p}<0.001$ vs control, !! $\mathrm{p}<0.01$ vs siNC. (B) SUM01 silencing promoted the TEER value in HTEC cells treated under hypoxic condition for $24 \mathrm{~h}$. *** $p<0.001$ vs control, !!! $p<0.001$ vs siNC. (C) SUMO-1 silencing reduced the permeability of HTEC cells treated under hypoxic condition for $24 \mathrm{~h} .{ }^{*} \mathrm{p}$ $<0.05$ vs control, $* * \star p<0.001$ vs siNC; !!! $p<0.001$ vs siNC. (D) Western blotting was used to examine the protein levels of SUM01, HIF-1a, VEGF, Occluding, Claudin-1 and ZO-1 in cells as indicated. 
A

HTEC

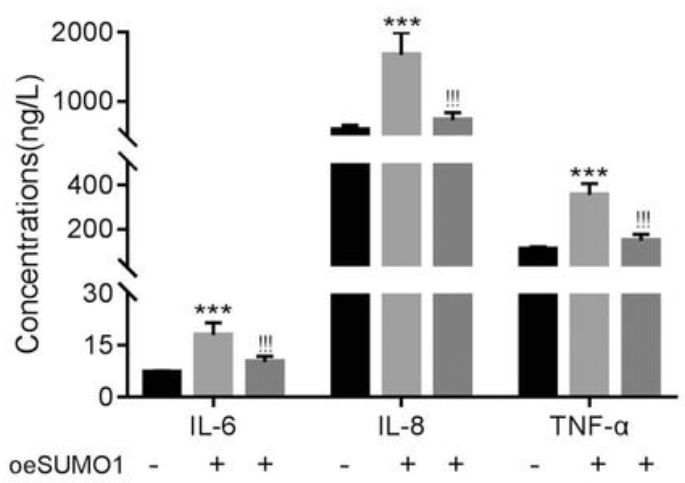

C

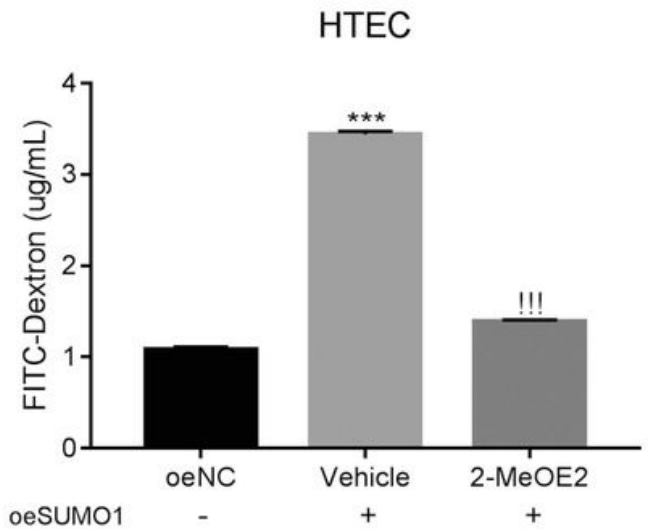

B

HTEC

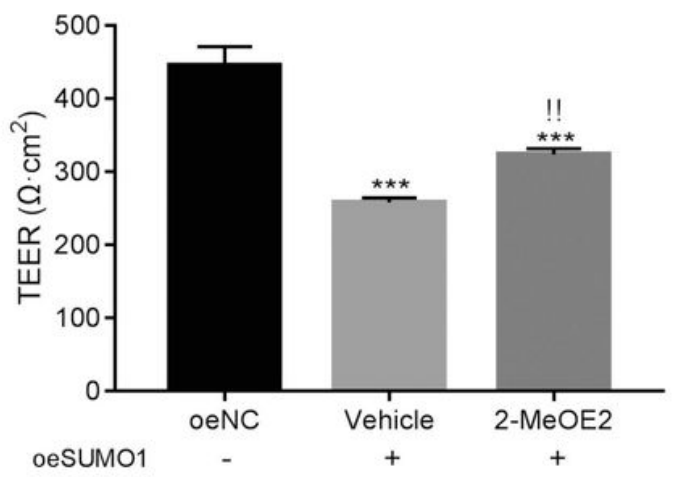

D

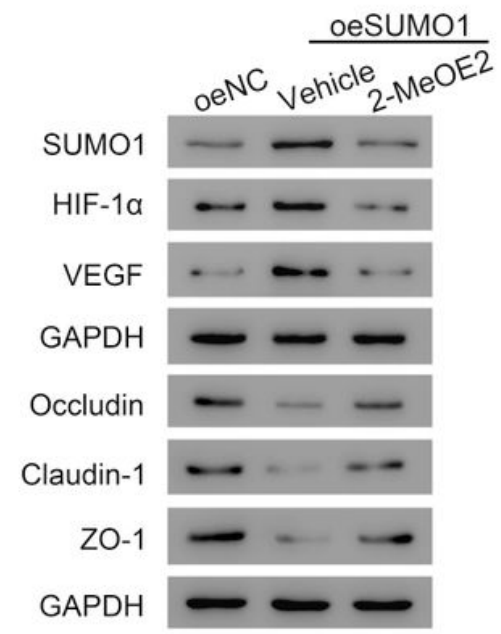

Figure 4

The HIF-1a inhibitor 2-MeOE2 suppresses the effects of SUMO-1 overexpression in HTEC cells. (A) 2MeOE2 suppressed the secretion of IL-6, IL-8 and TNF- $a$ in HTEC cells transfected with oeSUMO-1 vector. $\star \star \star ~ p<0.001$ vs oeNC, !!! $p<0.001$ vs oeSUM01+vehicle. (B) The TEER value was upregulated in cells transfected with oeSUMO1 vector in the presence of 2-MeOE2. *** $p<0.001$ vs oeNC, !! $p<0.01$ vs SUM01. (C) 2-MeOE2 decreased the permeability of HTEC cells transfected with oeSUMO1 vector. (D) Western blotting was used to examine the protein levels of SUMO-1, HIF-1a, VEGF, Occluding, Claudin-1 and ZO-1 in cells as indicated. 


\section{Cell lysate \\ $\lg G$ \\ SUMO1 Ab}

SUMO1

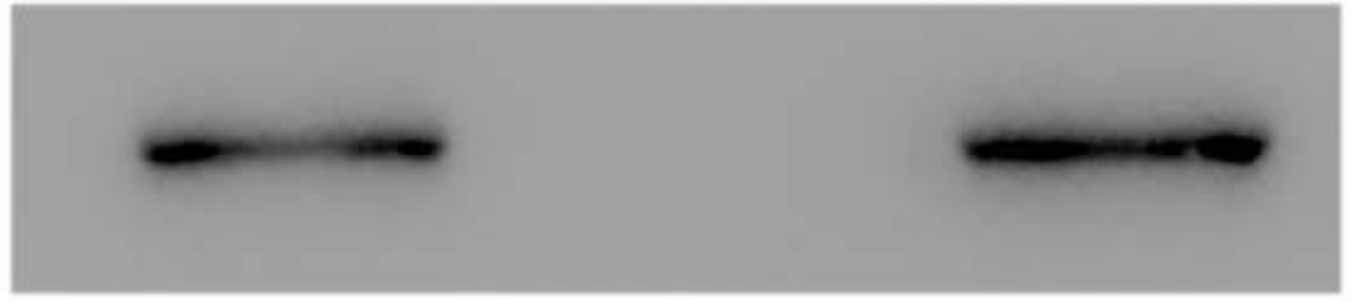

HIF-1a

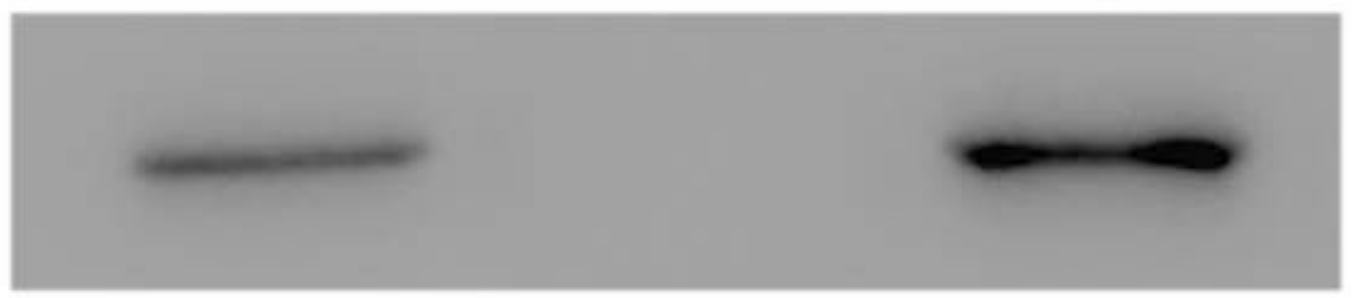

Cell lysate

$\lg G$

HIF-1 $1 a$ Ab

HIF-1a

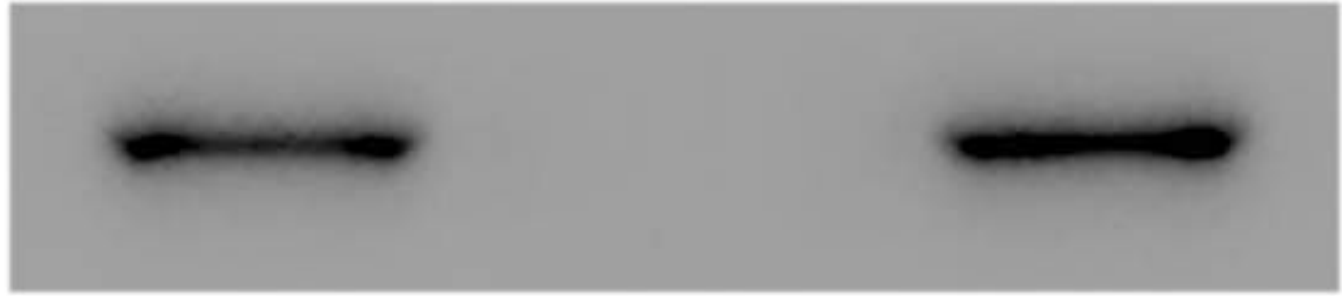

\section{SUMO1}

\section{Figure 5}

SUMO1 interacts with HIF-1a and regulates its ubiquitination in HTEC cells. (A) Co-IP assay was used to determine the interaction between SUMO-1 and HIF-1a in HTEC cells. (B) SUMO-1 silencing enhanced the ubiquitination of HIF-1a in HTEC cells. 
A

HTEC

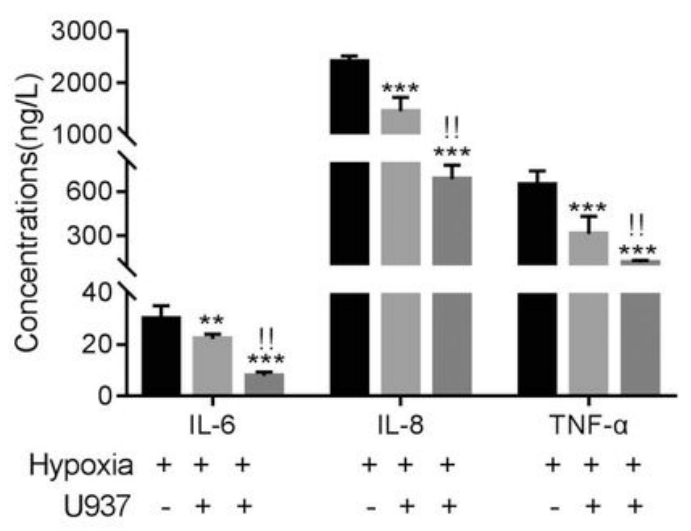

C

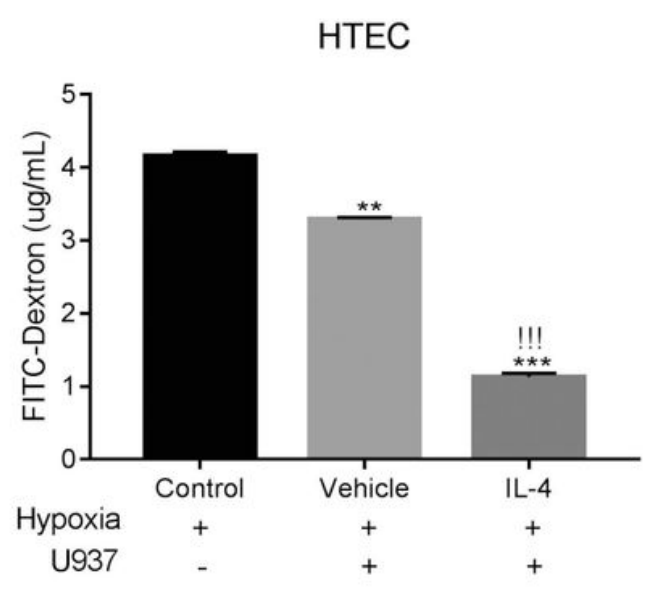

B

HTEC

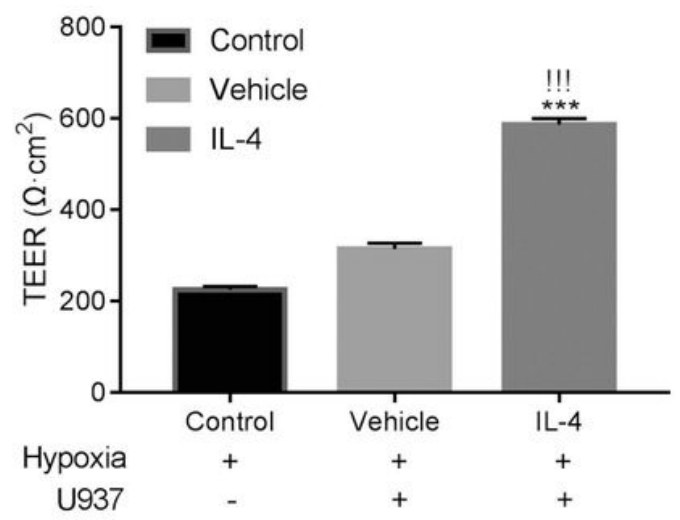

D

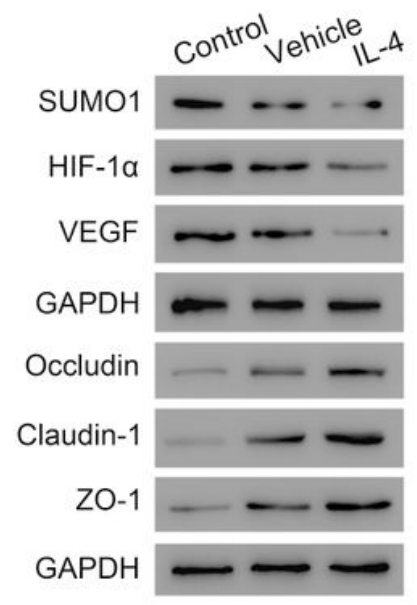

Figure 6

M2 type macrophages alleviate hypoxia-induced injury in HTEC cells. (A) The secretion of IL-6, IL-8 and TNF-a were decreased in HTEC cells co-cultured with M2 type macrophages under hypoxic condition. *** $p<0.001$ vs control, !! $p<0.01$ vs vehicle. (B) M2 type macrophages promoted the TEER value in HTEC cells under hypoxic condition. $* \star \star p<0.001$ vs control, !!! $p<0.001$ vs vehicle. (C) The permeability of HETC cells was decreased by co-culturing with M2 macrophages under hypoxic condition. ** $p<0.001$ vs control, ${ }^{\star \star \star} p<0.001$ vs control, !!! $p<0.001$ vs vehicle. (D) Western blotting was used to examine the protein levels of SUMO-1, HIF-1 a, VEGF, Occluding, Claudin-1 and ZO-1 in cells as indicated.

\section{Supplementary Files}

This is a list of supplementary files associated with this preprint. Click to download.

- FigureS1.jpg 